\title{
Reparação Vascular Após Implante de Stents Não-Farmacológicos e Farmacológicos em Modelo Experimental de Fibroateroma de Capa Fina em Coelhos
}

\author{
Dario Echeverri' ${ }^{1}$, K-Raman Purushothaman ${ }^{1}$, Pedro R. Moreno ${ }^{1}$
}

\section{RESUMO}

Fundamentos: A resposta de reparação vascular é um importante fator no desenvolvimento de reestenose e trombose no stent. O presente estudo foi planejado para avaliar a reparação vascular com stents não-farmacológicos (SNF) comparados a stents com liberação de everolimus (SLE) e de beta-estradiol (SLB) em um modelo experimental de fibroateroma de capa fina (FACF) em animais com aterosclerose crônica. Método: Foram analisados 16 coeIhos hipercolesterolêmicos da raça Nova Zelândia acompanhados por quatro anos. Desses animais, 6 receberam SNF, 5 receberam SLE e 5, SLB (Guidant, Santa Clara, Califórnia, Estados Unidos). Um stent com polímero também foi implantado em cada animal. Resultados: Análises histológicas realizadas aos 28 dias, comparando FACF de novo com FACF com implante de SNF, SLE e SLB, demonstraram que os stents com polímero induziram escores mais altos de fibrina e hemorragia. Os SLB induziram escores mais altos de inflamação e fibrina e escores mais baixos de endotelização. Os SLE induziram escores mais altos de inflamação, fibrina e hemorragia. SLB e SLE induziram escores de reparação semelhantes. A porcentagem das áreas de colágeno tipo I foi semelhante nos quatro tipos de stents. A porcentagem das áreas de colágeno tipo III foi mais alta com SNF quando comparados ao polímero e aos stents farmacológicos. Conclusão: Os stents farmacológicos estão associados a inflamação reduzida, mas crescente, deposição de fibrina e hemorragia, que parecem estar relacionadas aos efeitos vasculares do polímero.

DESCRITORES: Aterosclerose. Contenedores. Stents farmacológicos. Modelos animais. Coelhos.

${ }^{1}$ Gill Heart Institute, Universidade de Kentucky - Lexington, Kentucky, Estados Unidos.

Correspondência: Dario Echeverri. Servicio de Hemodinamia e Intervencionismo Cardiovascular. Jefe Laboratorio de Investigación en Función Vascular. Fundación Cardiolnfantil - Instituto de Cardiologia - Calle 163 A - número 13B-60 - Primer Piso - Bogotá, Colombia.

E-mail: decheverri@cardioinfantil.org funcionvascular@cardioinfantil.org

Recebido em: 12/9/2008 • Aceito em: 27/11/2008

\section{SUMMARY}

\author{
Vascular Healing After Bare Metal and \\ Drug-Eluting Stents in an Experimental Rabbit \\ Model of Thin Cap Fibroatheroma
}

Background: The vascular healing response is an important factor in the development of restenosis and stent thrombosis. This study was designed to evaluate the vascular healing of bare metal stents (BMS) compared to everolimus (EES) and beta-estradiol (BES) eluting stents in a chronic atherosclerotic experimental animal model of thin cap fibroatheroma (TCFA). Methods: Sixteen New Zealand hypercholesterolemic rabbits followed for 4 years were studied. Six animals received BMS, 5 EES, and 5 BES (Guidant, Santa Clara, CA). One polymer stent per animal was also implanted. Results: Histologic analysis at 28 days of de-novo vs. BMS, EES, and BES stented TCFA showed polymer stents induced a higher fibrin and hemorrhage score. BES induced higher inflammation and fibrin scores and a lower endothelization score. EES induced higher inflammation, fibrin and hemorrhage scores. BES and EES induced similar healing scores. Percent collagen I areas were similar in all 4 types of stents. Percent collagen III areas were higher in BMS when compared to polymer and drugeluting stents. Conclusion: Drug-eluting stents are associated with low but increased inflammation, fibrin deposition and hemorrhage which seem to be related to the polymer's vascular effects.

DESCRIPTORS: Atherosclerosis. Stents. Drug-eluting stents. Models, animal. Rabbits.

0 stents farmacológicos (SF) são o mais recente grande avanço tecnológico em intervenções coronárias percutâneas $(\mathrm{ICPS})^{1-5}$. As evidências acumuladas demonstram que, comparados aos stents não-farmacológicos (SNF), os SF são altamente eficazes para reduzir a reestenose angiográfica e a necessidade de repetição dos procedimentos de revascularização, e seus benefícios estendem-se também aos 
Echeverri D, et al. Reparação Vascular Após Implante de Stents Não-Farmacológicos e Farmacológicos em Modelo Experimental de Fibroateroma de Capa Fina em Coelhos. Rev Bras Cardiol Invas. 2008;16(4):474-481.

pacientes com características clínicas e angiográficas de alto risco $^{6-11}$.

Tratamentos eficazes são necessários para estabilizar placas ateroscleróticas não-estenóticas de alto risco, evitando complicações trombóticas da ruptura da placa. Meier \& Ramamurthy ${ }^{12}$ propuseram a ruptura intencional de lesões intermediárias com angioplastia com balão no momento do cateterismo. Esperava-se que a placa rota rica em lípides se transformasse em uma placa fibrótica e que o risco de um evento coronário fosse eliminado. Esse procedimento foi chamado de selamento da placa (plaque sealing) ${ }^{13}$. Desde então, abordagens percutâneas locais inovadoras têm sido propostas como tratamento das placas de alto risco ${ }^{14}$.

Os SNF são utilizados para estabilizar placas sintomáticas com rupturas e também podem estabilizar fibroateromas de capa fina (FACF) assintomáticos, sem ruptura, o tipo mais comum de placa aterosclerótica de alto risco ${ }^{15}$. No entanto, esse efeito benéfico é obtido à custa do espessamento neo-intimal induzido pelo stent, um mecanismo do tipo "faca de dois gumes", que também pode desencadear a reestenose. Além disso, o próprio stent pode romper a fina capa e induzir uma resposta mais agressiva ${ }^{16}$. Considerando que os SF têm potencial para reduzir o espessamento neo-intimal, eles podem combinar os efeitos estabilizadores dos SNF com risco reduzido de reestenose.

Os SF são uma opção de tratamento integral para pacientes com doença arterial coronariana. SF são projetados para liberar agentes farmacológicos na parede do vaso, para inibir a resposta ao dano vascular que causa a reestenose (migração de células musculares lisas vasculares, proliferação e remodelamento vascular). Entretanto, após o implante, essas substâncias exercem vários efeitos biológicos na circulação coronária, como migração e proliferação reduzidas das células endoteliais, indução de disfunção endotelial, interação com mediadores de transdução de sinal e aumento do potencial trombogênico dos SF. Por fim, o polímero utilizado pelos SF pode estar associado a reações de hipersensibilidade, que podem, pelo menos em alguns casos, favorecer a trombose no stent ${ }^{17}$. Esse efeito da reparação vascular é o novo calcanhar de Aquiles dos SF.

O presente estudo foi planejado para avaliar as vias da reparação vascular dos SNF comparados aos stents com liberação de everolimus (SLE) e de betaestradiol (SLB) em um modelo experimental de FACF, em animais com aterosclerose crônica.

\section{MÉTODO}

\section{Modelo experimental}

Conforme descrito anteriormente ${ }^{18}$, o Institutional Animal Care and Use Committee, da Universidade de Kentucky, Estados Unidos, aprovou o protocolo. Dezesseis coelhos brancos da raça Nova Zelândia foram colocados sob dieta suplementada com $1 \%$ de colesterol (Purina Mills Inc., Richmond, Indiana, Estados Unidos) por períodos de dois meses, alternados com dieta normal, por um total de oito meses, e acompanhados por um período mínimo de quatro anos.

\section{Protocolo experimental}

Foram implantados SLE em cinco animais, outros 5 receberam SLB e os 6 remanescentes receberam SNF (316L) balão-expansíveis (PENTA MULTILINK ${ }^{\mathrm{TM}}$ ) (Advanced Cardiovascular Systems, Santa Clara, Califórnia, Estados Unidos). No grupo de SLE, foi usado stent de liberação lenta (15\% liberados em 24 horas), com conteúdo médio do medicamento de $269 \mathrm{mg}$. No grupo de SLB, foi usado stent de liberação lenta (29\% liberados em 24 horas) com conteúdo médio do medicamento de $295 \mathrm{mg}$. Todos os stents tinham o mesmo comprimento $(8 \mathrm{~mm})$. Os stents receberam uma matriz de fluoropolímero. A matriz foi projetada para liberar cerca de $80 \%$ do fármaco nos trinta dias após o implante in vivo. Cada animal recebeu 4 stents seqüenciais na aorta abdominal. Além disso, um stent com polímero e um SNF foram implantados para comparação.

Todos os animais receberam $10 \mathrm{mg} / \mathrm{kg}$ de aspirina por via oral na noite anterior ao implante do stent. A indução da anestesia foi obtida com ketamina intravenosa $(20 \mathrm{mg} / \mathrm{kg}$ ) e xilazina $(4 \mathrm{mg} / \mathrm{kg})$, injetadas em veia marginal da orelha. A anestesia geral foi mantida com $2 \%$ de isoflurano e oxigênio, após intubação endotraqueal. A arteriotomia cirúrgica da artéria femoral comum direita com inserção de um introdutor $4 \mathrm{~F}$ foi seguida de anticoagulação com $100 \mathrm{UI} / \mathrm{kg}$ de heparina intravenosa. Um aortograma retrógrado distal foi realizado com injeção manual de contraste. Um fio de 0,014" foi introduzido sob fluoroscopia da artéria femoral até o segmento superior da aorta descendente, acima das artérias renais. Os stents foram implantados seqüencialmente no segmento distal da aorta abdominal, abaixo da artéria renal esquerda, com pressão de $14 \pm 2$ atm, obtendo-se razão stent/artéria de 1:1. Cuidado especial foi tomado para evitar o óstio da artéria lombar dentro do segmento tratado pelos stents. Após implante do stent foi realizado aortograma, para verificar se todos os stents foram implantados sem complicações. Ao final do procedimento, o introdutor foi removido e a artéria femoral foi ligada. Todos os animais foram observados durante a recuperação da anestesia e, então, devolvidos ao biotério. Após 28 dias de acompanhamento, foi realizada eutanásia eletiva com o uso de $150 \mathrm{mg} / \mathrm{kg}$ de pentobarbital intravenoso.

\section{Avaliação patológica}

Logo após a eutanásia, a aorta proximal foi canulada para permitir sua fixação com perfusão de formalina tamponada neutra a 10\%, com pressão de $60-80 \mathrm{mmHg}$ por trinta minutos. O segmento aórtico abaixo da artéria renal esquerda foi cuidadosamente dissecado, 
Echeverri D, et al. Reparação Vascular Após Implante de Stents Não-Farmacológicos e Farmacológicos em Modelo Experimental de Fibroateroma de Capa Fina em Coelhos. Rev Bras Cardiol Invas. 2008;16(4):474-481.

incrustado em metilmetacrilato e seccionado com lâmina de carboneto de aço inoxidável (Wasatch Histo Consultants, Winnemucca, Nevada, Estados Unidos), a cada $4 \mu \mathrm{m}$ a $5 \mu \mathrm{m}$, no caso das lesões com implante de stent (grupos experimentais). O segmento aórtico, desde a aorta torácica até a aorta abdominal, acima da artéria renal esquerda (aorta sem stent), foi incrustado em parafina e seccionado para obtenção de lesões de novo (grupo controle). Todas as seções histológicas foram coradas com hematoxilina e eosina (H\&E) e pelo método do tricrômio para elastina. O colágeno novo foi avaliado por coloração com vermelho pricosirius e microscopia de polarização ${ }^{19}$. Doze segmentos (3 segmentos por stent, 4 stents por animal) foram comparados a 20 segmentos de novo, sem stents, por animal.

\section{Morfometria}

A definição de FACF, aposição do stent, quantificação do dano vascular, análise da seção transversal e análise haste por haste foi publicada anteriormente ${ }^{18}$.
Reparação vascular: Quantificação detalhada da reparação vascular foi realizada para cada haste, segundo escores modificados adaptados de publicações anteriores ${ }^{20,21}$.

Inflamação: Definida como presença de células mononucleares em seções coradas com H\&E, utilizando objetiva com aumento de 40x e quantificada conforme mostra a Figura $1 \mathrm{~A}$.

Fibrina: Identificada por microscopia óptica como depósitos extracelulares amorfos (não-fibrilares) intensamente eosinofílicos sob coloração com H\&E e quantificada conforme a Figura 1B.

Hemorragia: Definida como extravasamento de coleções intersticiais de hemácias e macrófagos com pigmentação marrom (contendo hemossiderina) à microscopia óptica e quantificada conforme a Figura 1C.

Endotelização: Definida por microscopia óptica como presença de células endoteliais cobrindo as hastes e quantificada conforme a Figura 1D.


Figura 1 - Imagem composta mostrando exemplos de inflamação (A), deposição da fibrina (B), hemorragia (C) e endotelização (D), com escores I-IV; campo 40x. 
Echeverri D, et al. Reparação Vascular Após Implante de Stents Não-Farmacológicos e Farmacológicos em Modelo Experimental de Fibroateroma de Capa Fina em Coelhos. Rev Bras Cardiol Invas. 2008;16(4):474-481.

Colágeno: Identificado por microscopia de luz polarizada como matriz extracelular fibrilar entre as tonalidades amarela e vermelha (Tipo I) ou esverdeada (Tipo III) sob coloração com vermelho picrosirius, utilizando aumento de 20x, conforme mostra a Figura 2.

Quantificação da inflamação: Escore 0: sem inflamação ao redor da haste; Escore I: inflamação dispersa (< 25\% de deposição de células ao redor da haste); Escore II: inflamação cobrindo $25 \%$ a $50 \%$ da haste; Escore III: $50 \%$ a $75 \%$ de deposição ao redor da haste; e Escore IV: 100\% de deposição ao redor da haste.

Quantificação da fibrina: Escore 0: nenhuma fibrina ao redor da haste; Escore I: $<25 \%$ de deposição ao redor da haste; Escore II: $25 \%$ a $50 \%$ de deposição ao redor da haste; Escore III: 50\% a 75\% de deposição ao redor da haste; e Escore IV: 100\% de deposição ao redor da haste.

Quantificação da hemorragia: Escore 0: nenhuma hemácia ao redor da haste; Escore I: $<25 \%$ de deposição ao redor da haste; Escore II: 25\% a 50\% de deposição ao redor da haste; Escore III: 50\% a 75\% de deposição ao redor da haste; e Escore IV: 100\% de deposição ao redor da haste.

Quantificação da endotelização: Escore 0: nenhuma célula endotelial presente na haste; Escore I: < 25\% de deposição de células endoteliais ao redor da haste; Escore II: $25 \%$ a $75 \%$ da haste coberta por células endoteliais; Escore III: 100\% da haste coberta por células endoteliais; e Escore IV: haste coberta por tecido neo-intimal.

\section{Análise estatística}

Os dados referentes a SNF, SLE e SLB são apresentados utilizando-se média \pm erro padrão da média (EPM). Os dados que apresentaram distribuição nãogaussiana foram comparados utilizando-se o teste independente não-paramétrico $U$ de Mann-Whitney. Os dados que seguiram a distribuição gaussiana foram



Figura 2 - Imagem mostrando exemplos de seções de novo (sem implante de stent) e com implante de stent. A. Fibroateroma de capa fina (coloração pelo tricrômio para elastina; campo 20x). B. Fibroateroma de capa fina mostrando colágeno do Tipo I (microscopia de luz polarizada sob coloração vermelho picrosirius, de amarelo a vermelho; campo 20x). C. Seção com stent em fibroateroma de capa fina (coloração pelo tricrômio para elastina; campo 20x). D. Seção com stent em fibroateroma de capa fina mostrando matriz extracelular fibrilar, colágeno Tipo III (sob microscopia de luz polarizada em coloração vermelho picrosirius, aparece esverdeada; campo 20x). 
Echeverri D, et al. Reparação Vascular Após Implante de Stents Não-Farmacológicos e Farmacológicos em Modelo Experimental de Fibroateroma de Capa Fina em Coelhos. Rev Bras Cardiol Invas. 2008;16(4):474-481.

comparados com o teste $t$ de Student bicaudal. As variáveis dicotômicas foram comparadas com o teste do qui-quadrado. A significância foi estabelecida para um valor de $\mathrm{p} \leq$ 0,05. O software Statistical Package for Social Science (SPSS) 10.0.5 foi utilizado para as análises.

\section{RESULTADOS}

Os 16 coelhos avaliados neste estudo completaram o procedimento com sucesso, sem complicações graves. Anorexia, perda de peso, taquicardia e desidratação foram registradas em 4 coelhos, exigindo a administração de cristalóides por via intravenosa. Um dos coelhos recuperou-se, mas os outros 3 morreram em 4, 8 e 23 dias após o implante do stent. A necropsia identificou insuficiência renal aguda em dois coelhos e um grande infarto esplênico no terceiro coelho. Os 13 animais remanescentes foram submetidos a eutanásia eletiva 28 dias após o implante do stent. Dos 260 segmentos de novo avaliados, 76 demonstraram placas ateroscleróticas e foram considerados como grupo de novo. Além disso, dos 156 segmentos com stent avaliados, 138 apresentaram placas ateroscleróticas atrás das hastes e foram considerados como grupo com stent.

Conforme descrito na Tabela 1, em 237 hastes de SNF, stent com polímero e SF em FACF analisadas, houve aumento da área da capa fibrosa, detectada como nova capa fibrosa, nesse modelo experimental de aterosclerose avançada. Além disso, SLE e SLB estiveram associados a menor resposta neo-intimal.
Na análise haste a haste (campo 20x) com SF (SLB e SLE), não foram demonstradas mudanças significativas na área lipídica.

\section{Reparação vascular}

Os escores de reparação para os quatro tipos de stent são mostrados na Tabela 2. Foram observados baixos escores para inflamação, fibrina e hemorragia e escores elevados para endotelização, para todos os stents.

Em comparação aos SNF, os stents com polímero induzem escores mais elevados de fibrina e hemorragia ( $p<0,05$ para ambas as comparações) (Figura 3A). Além disso, os SLB induzem escores mais altos de inflamação e fibrina e escore mais baixo de endotelização quando comparados aos SNF ( $p \leq 0,05$ para todas as comparações) (Figura 3B). Os SLE também induzem escores mais altos de inflamação, fibrina e hemorragia quando comparados aos SNF ( $p \leq 0,05$ para todas as comparações) (Figura 3C). É interessante notar que tanto os SLB como os SLE induzem escores de reparação similares quando comparados aos stents com polímero ( $p=$ NS para todas as comparações) (Figura 3D).

Finalmente, a porcentagem das áreas do colágeno foi similar em todos os tipos de stents (SNF 4,7\% \pm $1,0 \%$, polímero $3,5 \% \pm 1,6 \%$, SLE 4,0\% $\pm 0,8 \%$, e SLB $4,3 \% \pm 0,6 \% ; p=$ NS para todas as comparações). No entanto, a porcentagem das áreas do colágeno III foi mais elevada nos SNF quando comparados aos stents com polímero e SF (SNF 60,5\% $\pm 30,6 \%$, polímero $17,0 \% \pm 6,0 \%$, SLE $26,1 \% \pm 5,4 \%$, e SLB $28,4 \% \pm$

TABELA 1

Histomorfometria por tipo de stent, em análise haste por haste

\begin{tabular}{lcccc}
\hline Análise haste por haste & $\begin{array}{c}\text { Não-farmacológico } \\
\mathbf{n}=\mathbf{1 2 7}\end{array}$ & $\begin{array}{c}\text { Polímero } \\
\mathbf{n = 2 3}\end{array}$ & $\begin{array}{c}\text { Beta-estradiol } \\
\mathbf{n}=\mathbf{4 6}\end{array}$ & $\begin{array}{c}\text { Everolimus } \\
\mathbf{n}=\mathbf{4 1}\end{array}$ \\
\hline Área de lípides $\left(\mathrm{mm}^{2}\right)$ & $0,39 \pm 0,2$ & $0,39 \pm 0,5$ & $0,39 \pm 0,4$ & $0,37 \pm 0,3$ \\
Área da capa fibrosa antiga $\left(\mathrm{mm}^{2}\right)$ & $0,26 \pm 0,1$ & $0,37 \pm 0,4^{*}$ & $0,24 \pm 0,2$ & $0,23 \pm 0,2$ \\
Área da nova capa fibrosa $\left(\mathrm{mm}^{2}\right)$ & $1,05 \pm 0,4$ & $1,1 \pm 0,6$ & $0,88 \pm 0,7^{* *}$ & $0,73 \pm 0,4^{* *}$ \\
\hline
\end{tabular}

* $\mathrm{p}<0,05$ em comparação com o grupo não-farmacológico; ** $p<0,05$ em comparação com o grupo polímero.

TABELA 2

Reparação vascular por tipo de stent, em análise haste por haste

\begin{tabular}{lcccc} 
Reparação vascular & $\begin{array}{c}\text { Não-farmacológico } \\
\mathbf{n}=\mathbf{1 2 7}\end{array}$ & $\begin{array}{c}\text { Polímero } \\
\mathbf{n = 2 3}\end{array}$ & $\begin{array}{c}\text { Beta-estradiol } \\
\mathbf{n = 4 6}\end{array}$ & $\begin{array}{c}\text { Everolimus } \\
\mathbf{n}=\mathbf{4 1}\end{array}$ \\
\hline Quantificação da inflamação & $0,9 \pm 0,1$ & $1,0 \pm 0,1$ & $1,1 \pm 0,1^{* *}$ & $1,1 \pm 0,1^{* *}$ \\
Quantificação da fibrina & $0,6 \pm 0,1$ & $1,1 \pm 0,2^{*}$ & $1,1 \pm 0,1^{* *}$ & $1,1 \pm 0,2^{* *}$ \\
Quantificação da hemorragia & $0,1 \pm 0,0$ & $0,2 \pm 0,1^{*}$ & $0,1 \pm 0,0$ & $0,2 \pm 0,1^{* *}$ \\
Quantificação da endotelização & $3,7 \pm 0,1$ & $3,7 \pm 0,1$ & $3,2 \pm 0,2^{* *}$ & $3,6 \pm 0,1$ \\
\hline
\end{tabular}

* p $<0,05$ em comparação com o grupo não-farmacológico; ${ }^{* *} \mathrm{p}<0,05$ em comparação com o grupo polímero. 
A

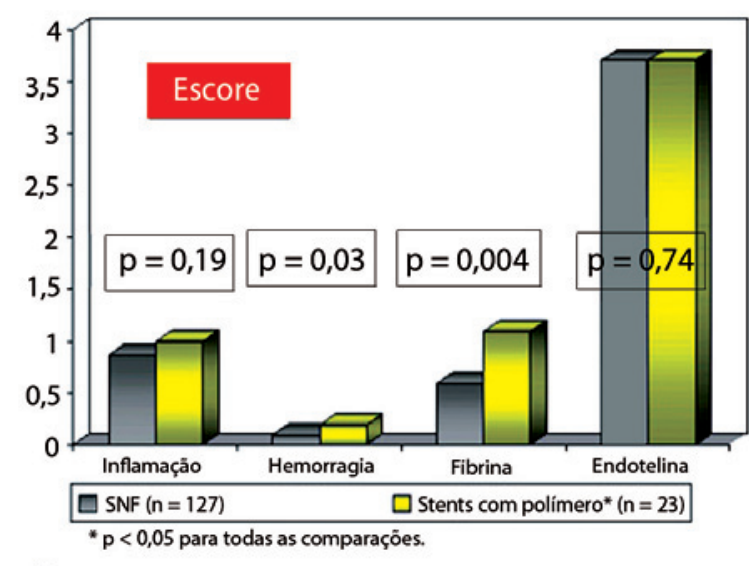

C

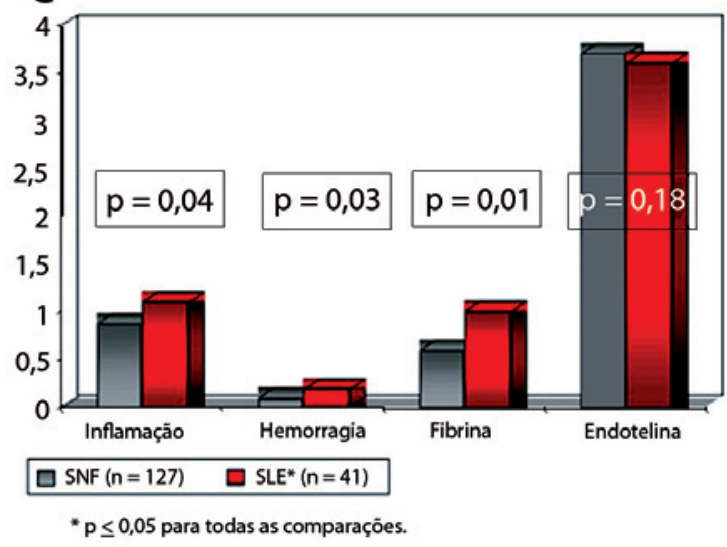

B
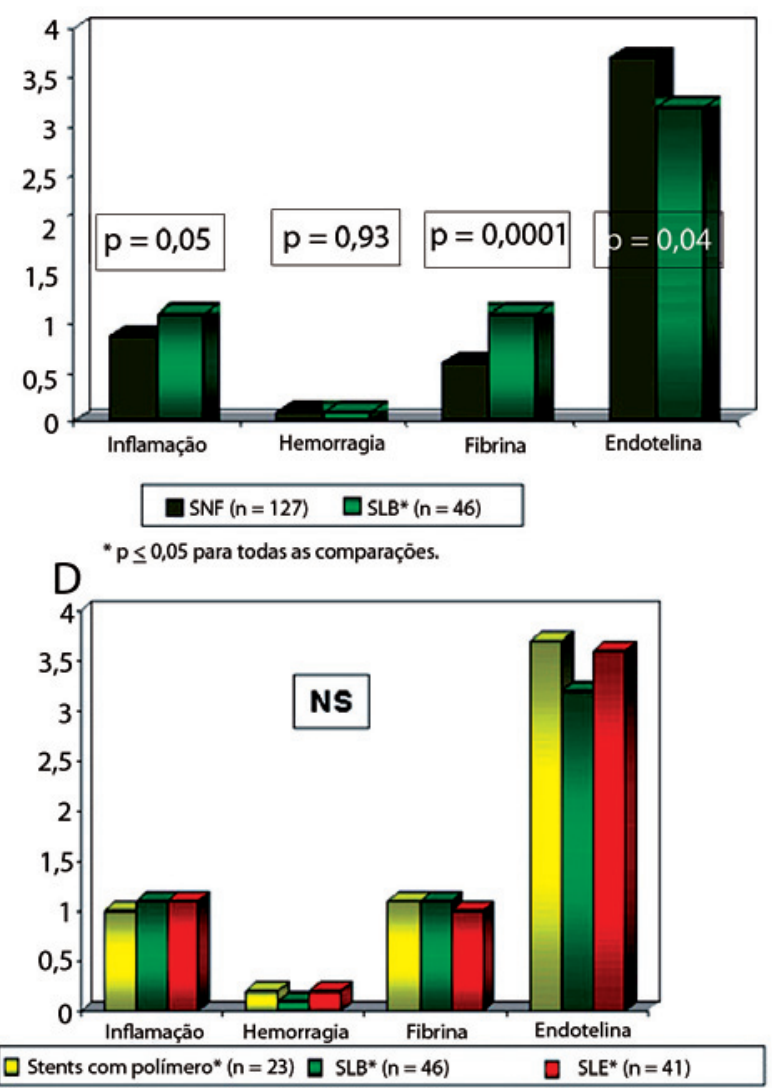

${ }^{*} \mathrm{p}=\mathrm{NS}$ para todas as comparações.

Figura 3 - Imagem composta mostrando comparações de escores da reparação vascular entre os stents. O eixo vertical (Y) mostra a gravidade na escala, de 0 a 4 . NS = não-significante; SLB = stents com liberação de beta-estradiol; SLE = stents com liberação de everolimus; SNF = stents não-farmacológicos.

3,0\%; $\mathrm{p} \leq 0,05$ para todas as comparações). Quando comparados aos stents com polímero, tanto os SLE como os SLB induzem porcentagem similar de áreas do colágeno III ( $p=N S$ ).

\section{DISCUSSÃO}

A resposta normal de reparação da parede arterial ao dano que ocorre após implante do stent coronariano compreende dois processos principais: a formação de uma neo-íntima (íntima nova) e a reendotelização da superfície vascular lesada ${ }^{22}$. Este estudo avaliou pela primeira vez as respostas biológicas ao implante de stent em um modelo experimental de FACF em coeIhos com aterosclerose avançada. Relatamos anteriormente $^{18}$ que o implante de stent em FACF aumenta a espessura da capa fibrosa e reduz o conteúdo lipídico. Além disso, SLE e SLB estão associados à resposta neo-intimal controlada.

Neste estudo experimental, a reparação vascular demonstrou escores baixos para inflamação, fibrina e hemorragia com SF; a endotelização mostrou escores elevados para todos os stents, com maior porcentagem das áreas de colágeno I e menor porcentagem das áreas do colágeno III. É interessante notar que tanto SLB como SLE induzem escores de reparação similares quando comparados aos stents com polímero. Além disso, efeitos de reparação similares foram observados com polímero e SF, sugerindo que as diferenças quanto a inflamação, deposição de fibrina e hemorragia entre SNF e SF talvez estejam relacionadas ao componente do polímero e não ao medicamento ativo encontrado nos SLB e SLE.

Apesar de recentes avanços, a reestenose intrastent (RIS) continua sendo um desafio para a cardiologia intervencionista, com taxa global estimada de reestenose de $20 \%$ a aproximadamente $25 \%$ em SNF e de cerca de $12 \%$ em SF. O trabalho experimental em animais e o estudo histológico de stents coronarianos humanos sugerem que angioplastia com balão ou implante de stent sejam responsáveis por uma resposta inflama- 
Echeverri D, et al. Reparação Vascular Após Implante de Stents Não-Farmacológicos e Farmacológicos em Modelo Experimental de Fibroateroma de Capa Fina em Coelhos. Rev Bras Cardiol Invas. 2008;16(4):474-481.

tória seguida por crescimento neo-intimal. Em modelos animais, a lesão arterial após angioplastia com balão tem sido associada a níveis locais elevados de marcadores da inflamação ${ }^{23-27}$ e a crescimento da neoíntima ${ }^{28}$. O surgimento dos SF representou real avanço na prevenção e no tratamento da reestenose, porém os agentes antimitóticos presentes são relativamente inespecíficos e podem levar a eventos adversos em regiões circunjacentes ${ }^{29,30}$ e a processo prolongado de cicatrização. O processo de RIS é paralelo à resposta de cicatrização da ferida. A gravidade da lesão arterial durante o implante de stent correlaciona-se com inflamação elevada e crescimento neo-intimal tardio ${ }^{31}$.

Os medicamentos liberados pelos SF exercem efeitos biológicos distintos, como ativação das vias de transdução de sinal e inibição da proliferação celular. Como resultado, embora inicialmente visem a evitar a proliferação e migração das células musculares lisas vasculares (um dos principais fatores para desenvolvimento de reestenose), prejudicam, também, a reendotelização, o que leva à reparação arterial tardia e induz a expressão do fator tecidual, resultando em um ambiente pró-trombogênico ${ }^{32}$.

Embora rara, a trombose no stent continua sendo uma complicação grave após o implante do stent, em decorrência de sua elevada morbidade e mortalidade. Alguns dados de grandes registros e meta-análises de estudos randomizados indicam risco mais elevado de trombose nos SF, enquanto outros sugerem a ausência de tal risco. Vários fatores estão associados ao risco elevado de trombose no stent, incluindo o próprio procedimento (má aposição do stent, subexpansão, número de stents implantados, tamanho do stent, fluxo sanguíneo coronário lento persistente e dissecções), características do paciente e das lesões, modelo do stent e interrupção prematura dos medicamentos antiplaquetários. Estudos anteriores com SF em animais descreveram diferentes graus de reparação vascular, conforme relatado por Virmani et al. ${ }^{33}$. O implante permanente de stent pode provocar reações granulomatosas vasculares que podem afetar as respostas de reparação tardia e os resultados $\operatorname{clínicos}^{34}$.

Embora o polímero possa ter efeito na vasculatura coronária após o implante de SF, a relação causal entre inflamação induzida pelo polímero e incidência de trombose tardia no stent foi sugerida apenas em uma minoria de pacientes ${ }^{35}$. A utilização de um polímero biocompatível, a fosforilcolina, demonstrou in vitro ser resistente à adsorção do fibrinogênio e causar menor ativação das plaquetas e monócitos ${ }^{36}$. Além disso, sua rápida cinética de liberação do medicamento também poderia reduzir a toxicidade vascular local ${ }^{37}$.

O desempenho dos SF não deve se basear apenas no grau da supressão neo-intimal, mas sim na resposta celular a esses dispositivos, em especial no tocante à reparação arterial. Precisamos conseguir maior equi- líbrio entre essas duas propostas ambiciosas. A curto prazo, isso provavelmente significa criar dispositivos que apresentem maior perda tardia (e, portanto, que incentivem maior cobertura da haste pela neo-íntima). A longo prazo, isso pode significar a utilização de agentes antiproliferativos específicos, que atuem no músculo liso, sem afetar a regeneração endotelial ${ }^{38}$.

As estratégias atuais para reduzir a incidência de RIS após intervenção coronária percutânea estão direcionadas para modificar a reparação arterial após lesão no stent. Diferentes estratégias estão sendo desenvolvidas para obter SF com melhor sistema de liberação, eficácia e segurança. Muitas dessas estratégias visam à redução do papel da reparação vascular como possível mecanismo que favorece a trombose no stent e incluem novos modelos de stents e plataformas, redução das hastes de stent utilizando uma liga de cobalto-cromo, combinação de medicamentos antiinflamatórios e antiproliferativos, melhores polímeros e novos sistemas de liberação de medicamentos. Os critérios de desempenho mais importantes para os novos agentes de SF incluem maior especificidade do tipo celular, margens de segurança mais amplas e maior facilidade para promover endotelização e reparação ${ }^{39}$. Essas recentes estratégias de reparação merecem ser mais bem exploradas, pois podem representar uma alternativa para abordar os $\mathrm{SF}^{40}$.

\section{CONCLUSÃO}

O implante de stent em FACF aumenta a espessura da capa fibrosa e reduz a área lipídica em modelo experimental de aterosclerose crônica avançada. Contudo, os SF estão associados a inflamação reduzida, mas crescente, deposição de fibrina e hemorragia, que parecem estar relacionadas ao polímero. A reparação arterial após implante de SF é multifatorial. Com a nova geração de SF, é extremamente importante que fatores como endotelização, inflamação, cinética da liberação e redução neo-intimal sejam examinados em testes pré-clínicos de modelos de animais para estabelecer a segurança desses dispositivos em humanos.

\section{AGRADECIMENTOS}

Os autores agradecem a Deborah Kilpatrick, PhD, William N. O'Connor, MD, e aos doutores Randy Stevens, Ken Dickey, Mark Mathew e Gentian Kristo, por sua ajuda durante os procedimentos experimentais, e ao sr. Terry Lacy, pela perícia em histotecnologia. Os autores expressam também seu reconhecimento ao The Vulnerable Plaque Team, da Guidant Corporation, pelo patrocínio e apoio diligente em todas as fases do estudo.

\section{REFERÊNCIAS BIBLIOGRÁFICAS}

1. Faxon DP. Bringing reality to drug-eluting stents. Circulation. $2004 ; 109(2): 140-2$. 
Echeverri D, et al. Reparação Vascular Após Implante de Stents Não-Farmacológicos e Farmacológicos em Modelo Experimental de Fibroateroma de Capa Fina em Coelhos. Rev Bras Cardiol Invas. 2008;16(4):474-481.

2. Sousa JE, Serruys PW, Costa MA. New frontiers in cardiology: drug-eluting stents: part I. Circulation. 2003;107(17): 2274-9.

3. Bauters C, Isner JM. The biology of restenosis. Prog Cardiovasc Dis. 1997;40(2):107-16.

4. Sousa JE, Serruys PW, Costa MA. New frontiers in cardiology: drug-eluting stents: part II. Circulation. 2003;107(18): 2383-9.

5. Muni NI, Gross TP. Problems with drug-eluting coronary stents: the FDA perspective. N Engl J Med. 2004;351(16):1593-5.

6. Morice MC, Serruys PW, Sousa JE, Fajadet J, Ban Hayashi E, Perin $M$, et al. A randomized comparison of a sirolimuseluting stent with a standard stent for coronary revascularization. RAVEL Study Group. N Engl J Med. 2002;346(23): 1773-80.

7. Moses JW, Leon MB, Popma JJ, Fitzgerald PJ, Holmes DR, O'Shaughnessy C, et al., SIRIUS Investigators. Sirolimuseluting stents versus standard stents in patients with stenosis in a native coronary artery. N Engl J Med. 2003;349(14): 1315-23.

8. Colombo A, Drzewiecki J, Banning A, Grube E, Hauptmann $\mathrm{K}$, Silber $\mathrm{S}$, et al. Randomized study to assess the effectiveness of slow- and moderate release polymer-based paclitaxeleluting stents for coronary artery lesions. TAXUS II Study Group. Circulation. 2003;108(7):788-94.

9. Stone GW, Ellis SG, Cox DA, Hermiller J, O'Shaughnessy C, Mann JT, et al. A polymer-based, paclitaxel-eluting stent in patients with coronary artery disease. TAXUS IV Investigators. N Engl J Med. 2004;350(3):221-31.

10. Ardissino D, Cavallini C, Bramucci E, Indolfi C, Marzocchi A, Manari A, et al. Sirolimus-eluting vs. uncoated stents for prevention of restenosis in small coronary arteries: a randomized trial. SES-SMART Investigators. JAMA. 2004;292(22): 2727-34.

11. Stone GW, Ellis SG, Cannon L, Mann JT, Greenberg JD, Spriggs D, et al. Comparison of a polymer-based paclitaxeleluting stent with a bare metal stent in patients with complex coronary artery disease: a randomized controlled trial. TAXUS IV Investigators. JAMA. 2005;294(10):1215-23.

12. Meier $B$, Ramamurthy $S$. Plaque sealing by coronary angioplasty. Cathet Cardiovasc Diagn. 1995;36(4):295-7.

13. Meier B. Plaque sealing by coronary angioplasty. Heart. 2004;90(12):1395-8.

14. Demidova TN, Hamblin MR. Photodynamic therapy targeted to pathogens. Int J Immunopathol Pharmacol. 2004; 17(3):245-54.

15. Kolodgie FD, Burke AP, Farb A, Gold HK, Yuan J, Narula J, et al. The thin-cap fibroatheroma: a type of vulnerable plaque: the major precursor lesion to acute coronary syndromes. Curr Opin Cardiol. 2001;16(5):285-92.

16. Farb A, Weber DK, Kolodgie FD, Burke AP, Virmani R. Morphological predictors of restenosis after coronary stenting in humans. Circulation. 2002;105(25):2974-80.

17. Steffel J, Tanner FC. Biological effects of drug-eluting stents in the coronary circulation. Herz. 2007;32(4):268-73.

18. Echeverri D, Purushothaman KR, Kilpatrick D, O'Connor WN, Moreno PR. Estabilização da placa aterosclerótica por stents farmacológicos e não-farmacológicos em modelo experimental de fibroateroma de capa fina em coelhos. Rev Bras Cardiol Invas. 2008;16(2):170-7.

19. Junqueira LC, Bignolas G, Brentani RR. Picrosirius staining plus polarization microscopy, a specific method for collagen detection in tissue sections. Histochem J. 1979;11(4):447-55.

20. Kornowski R, Hong MK, Tio FO, Bramwell $\mathrm{O}, \mathrm{Wu} \mathrm{H}$, Leon $M B$. In-stent restenosis: contributions of inflammatory responses and arterial injury to neointimal hyperplasia. J Am Coll Cardiol. 1998;31(1):224-30.
21. Farb A, Weber DK, Kolodgie FD, Burke AP, Virmani R. Morphological predictors of restenosis after coronary stenting in humans. Circulation. 2002;105(25):2974-80.

22. Costa MA, Simon DI. Molecular basis of restenosis and drug-eluting stents. Circulation. 2005;111(17):2257-73.

23. Kornowski R, Hong MK, Tio FO, Bramwell O, Wu H, Leon $M B$. In-stent restenosis: contributions of inflammatory responses and arterial injury to neointimal hyperplasia. J Am Coll Cardiol. 1998;31(1):224-30.

24. Hayashi S, Watanabe N, Nakazawa K, Suzuki J, Tsushima K, Tamatani $\mathrm{T}$, et al. Roles of P-selectin in inflammation, neointimal formation, and vascular remodeling in balloon-injured rat carotid arteries. Circulation. 2000;102(14):1710-7.

25. Okamoto E, Couse T, De Leon H, Suzuki J, Tsushima K, Tamatani T, et al. Perivascular inflammation after balloon angioplasty of porcine coronary arteries. Circulation. 2001; 104(18):2228-35.

26. Breuss JM, Cejna M, Bergmeister $H$, Kadl A, Baumgartl G, Steurer S, et al. Activation of nuclear factor-kappa B significantly contributes to lumen loss in a rabbit iliac artery balloon angioplasty model. Circulation. 2002;105(5):633-8.

27. Zhou Z, Lauer MA, Wang K, Forudi F, Zhou X, Song X, et al. Effect of anti-tumor necrosis factor-alpha polyclonal antibody on restenosis after balloon angioplasty in a rabbit atherosclerotic model. Atherosclerosis. 2002;161(1):153-9.

28. Farb A, Weber DK, Kolodgie FD, Burke AP, Virmani R. Morphological predictors of restenosis after coronary stenting in humans. Circulation. 2002;105(25):2974-80.

29. Bhatia V, Bhatia R, Dhindsa M. Drug-eluting stents: new era and new concerns. Postgrad Med J. 2004;80(939):13-8.

30. Marx SO, Marks AR. Bench to bedside: the development of rapamycin and its application to stent restenosis. Circulation. 2001;104(8):852-5.

31. Virmani R, Farb A. Pathology of in-stent restenosis. Curr Opin Lipidol. 1999;10(6):499-506.

32. Lüscher TL, Steffel J, Eberli FR, Joner M, Nakazawa G, Tanner FC, et al. Drug-eluting stent and coronary thrombosis: biological mechanisms and clinical implications. Circulation. 2007;115(8):1051-8.

33. Virmani R, Kolodgie FD, Farb A, Lafont A. Drug eluting stents: are human and animal studies comparable? Heart. 2003;89(2):133-8.

34. Kornowski R, Hong MK, Virmani R, Jones R, Vodovotz $\mathrm{Y}$, Leon MB. Granulomatous "foreign body reactions" contribute to exaggerate in-stent restenosis. Coron Artery Dis. 1999;10(1):9-14

35. Burke AP, Kolodgie FD, Farb A, Weber DK, Malcom GT, Smialek J, et al. Healed plaque ruptures and sudden coronary death evidence that subclinical rupture has a role in plaque progression. Circulation. 2001;103(7):934-40.

36. Whelan DM, van der Giessen WJ, Krabbendam SC, van Vliet EA, Verdouw PD, Serruys PW, et al. Biocompatibility of phosphorylcholine coated stents in normal porcine coronary arteries. Heart. 2000;83(3):338-45.

37. Kandzari DE, Leon MB. Overview of pharmacology and clinical trials program with the zotarolimus-eluting endeavor stent. J Interv Cardiol. 2006;19(5):405-13.

38. Finn AV, Nakazawa G, Joner $M$, et al. Vascular responses to drug eluting stents: importance of delayed healing. Arterioscler Thromb Vasc Biol. 2007;27(7):1500-10.

39. Ako J, Bonneau HN, Honda Y, Fitzgerald PJ. Design criteria for the ideal drug-eluting stent. Am J Cardiol. 2007;100(8B): $3 \mathrm{M}-9 \mathrm{M}$.

40. Noordeloos AM, Soullié T, Duckers HJ, Serruys PW. Promoting vascular regeneration as an alternative to conventional angioplasty-based intervention. Endothelium. 2006;13(6):431-9. 